\title{
Multimodal Location Estimation of Consumer Media: Dealing with Sparse Training Data
}

\author{
Jaeyoung Choi, Gerald Friedland \\ International Computer Science Institute \\ 1947 Center St., Berkeley, CA, USA \\ \{jaeyoung, fractor\}@icsi.berkeley.edu
}

\author{
Venkatesan Ekambaram, Kannan Ramchandran \\ Department of EECS, UC Berkeley \\ Berkeley, CA, USA \\ \{venkyne,kannanr\}@eecs.berkeley.edu
}

\begin{abstract}
This article describes a novel approach to the problem of associating geo-locations to consumer-produced multimedia data such as videos and photos that are publicly available on social networking websites such as Flickr. We specifically focus on the case where the available training data is sparse both in absolute numbers as well as geographic coverage when compared to the number of untagged query data. We develop a novel graphical model based framework for the problem of interest and pose the problem of geotagging as one of inference over this graph. The novelty of our algorithm lies in the fact that we jointly estimate the geo-locations of all the query videos, which helps obtain performance improvements over existing algorithms in the literature that process each query video independently. Our system enables the query videos to act as "virtual" training data that effectively bootstrap the geo-tagging process. The quality of the database improves with each additional query video in the system. Further, our modeling provides a generic theoretical framework that can be used to incorporate any other available textual, visual or audio features. We evaluate our algorithm on the MediaEval 2011 Placing Task data set and show that for fixed training data the system performance improves with an increasing number of unlabeled test data. The performance gains are shown to be over $10 \%$ as compared to existing algorithms in the literature.
\end{abstract}

Keywords-Multimodal Location Estimation, Geo-Tagging, Graphical Models, Belief Propagation.

\section{INTRODUCTION}

With the emergence of Web 2.0 and with GPS devices becoming ubiquitous and pervasive in our daily life, location-based services are rapidly gaining traction in the online world. The main driving force behind these services is the enabling of a very personalized experience. Socialmedia websites such as Flickr, YouTube, Twitter, etc., allow queries for results originating at a certain location. Likewise, the belief is that retro-fitting archives with location information will be attractive to many businesses, and will enable newer applications. The task of estimating the geo-coordinates of a media-recording goes by different names such as "geo-tagging", "location estimation" or "placing". Geo-tagging multimedia content has various applications. For example, geo-location services can be provided for media captured in environments without GPS, such as photos taken indoors on mobile phones. Vacation videos and photos can be better organized and presented

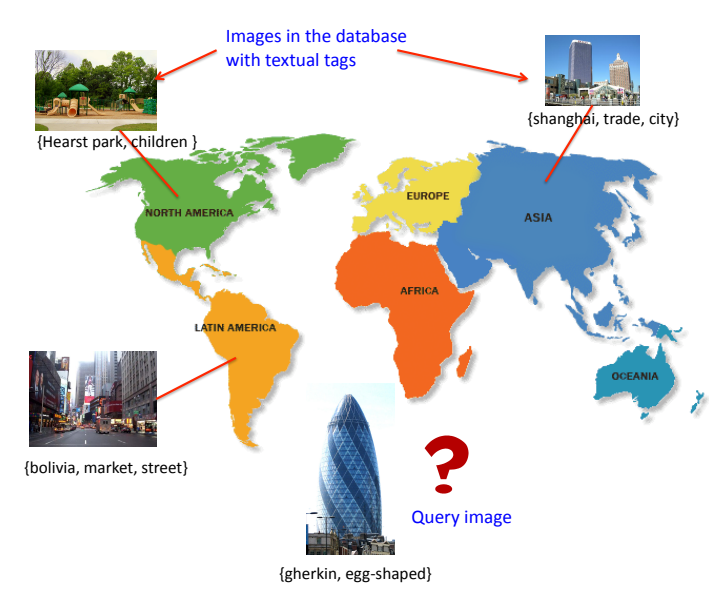

Figure 1. Geo-tagging: given a database of training images/videos with their geo-coordinates and textual data, estimate the geo-location of a query video given its textual metadata, visual and audio features.

to the user if they have geo-location information. With the explosive growth of available multimedia content on the Internet (200 million photos are uploaded to Facebook daily), there is a dire need for efficient organization and retrieval of multimedia content, which can be enabled by geo-tagging. Geo-location information further helps develop a better semantic understanding of multimedia content. These were some of the main motivations of the MediaEval Placing task [1], [2].

Even though many of the high-end cameras and video recorders are retrofitted with GPS chips, it has been estimated that only about $5 \%$ of the existing multimedia content on the Internet is actually geo-tagged [3]. Most of the consumer-produced media content are obtained using low-end cameras that do not have GPS chips. Further, privacy concerns have motivated users to disable automatic geo-stamping of photos taken on their phones. However, users usually tag their uploaded videos with textual data that can have some geo-location information. Under this scenario, we ask the question, "Given a set of videos and their associated textual tags, how do we determine their geo-locations?" To aid this process, we are provided with a training database of videos with their geo-location estimates. 
The main contribution of our work is the development of an estimation theoretic collaborative framework based on graphical models for the purpose of determining the geo-coordinates of query videos. We pose the problem of geo-tagging as one of inference over an underlying graph constructed using the query video database and jointly estimate the geo-locations of all the query videos. The main advantage of this approach is that, even when the training data is sparse, the query videos whose geo-tags are estimated act as "virtual" training data for incoming query data, thereby effectively bootstrapping the geo-tagging process. Our results show that we obtain up to $10 \%$ performance improvements over existing state-of-the-art algorithms. The generic nature of the framework facilitates fusing multimodal data sources such as textual tags, audiovisual features, etc. Thus, any other additional information obtained can be easily incorporated into the framework.

Our paper is organized as follows. Section II provides a brief overview of the existing work in this field and the novelty of our work as compared to this literature. Section III describes in detail the problem of data sparsity and a simple example illustrating the intuition behind our algorithm. Section IV describes in detail our graphical model framework for geo-tagging. Section V describes the datasets we use and our simulation results. Section VI concludes with a summary of the paper and possible research directions.

\section{LITERATURE REVIEW}

The problem of geo-tagging is closely related to the problem of user location estimation using images captured from his/her camera. Some of the earlier work in this domain [4], [5] posed the problem as one of image retrieval from a database of self-produced, location-tagged images using specialized visual features. Similar approaches based on visual descriptors such as [6] have been used to geo-tag images. Larson et al. [7] provide a comprehensive overview of the ongoing research in this field. Most users tag the media they upload to sites like Flickr with text that can include information regarding the location or activity captured by the images. Rattenbury et al. [8] and Serdyukov et al. [9] estimate the posterior distribution of the geo-locations given the tags or vice-versa from the training database and use this to estimate the geo-location of a query video.

The 2010 and 2011 MediaEval Placing tasks [1] provided a common platform to evaluate different geo-tagging approaches on a corpus of randomly selected consumerproduced videos. One of the top performing systems proposed by Van Laere et al. [10] used a combination of language models and similarity search to geo-tag the videos purely based on their textual tags. Several other proposed approaches [11]-[14] relied on both textual and visual

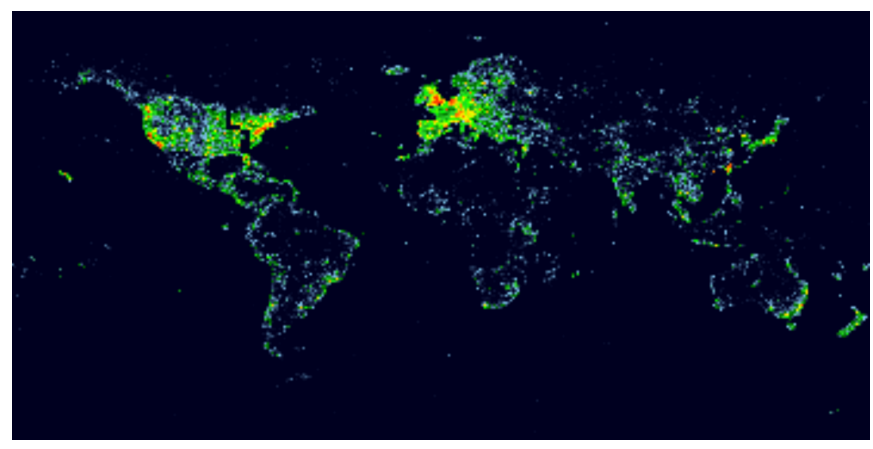

Figure 2. Distribution of videos and images of the MediaEval 2010 Placing Task training set. Randomly sampling videos from Flickr results in a nonuniform geographical prior.

features. However, none of these systems utilized audio features. A subset of the authors of this paper proposed a hierarchical system [15] that uses the spatial variance of the tags' geo-location distribution to find an initial estimate of the query image location, which is used as an anchor point for a visual nearest neighbor search in the next stage. Their enhanced system [16] incorporates audio features as well, motivated by their previous work on location estimation of ambulance videos from different cities [3] using audio features.

All of the existing approaches described above have the common feature of processing each query video independently and estimating its geo-location based on textual, visual, and audio features using a geo-tagged training database. Clearly, the performance of these systems largely depends on the size and quality of the training database. However, data sparsity is one of the major issues that can adversely affect the performance of these systems. Our approach differs from the existing work in the literature in the aspect that we jointly estimate the geo-locations of all the input query images. Each query image added to the database enhances the quality of the database by acting as "virtual" training data and thereby boosts the performance of the algorithm. We elaborate on the data sparsity issue in the next section and provide an intuition for our algorithm.

\section{DATA SPARSITY}

Traditional approaches such as [12], [15] use training sets that are several orders of magnitude larger than the test set. These approaches suffer from the drawback that their accuracies are significantly affected when the training data is sparse. There are two reasons for sparsity in training data. First, it is estimated that only $5 \%$ of Internet videos are actually geo-tagged [3], and hence the training set is typically much smaller than the test set, contrary to what is assumed in the literature. Second, the training database is largely skewed toward certain geographical regions (see Fig. 2).

For the MediaEval dataset [1], we analyzed the performance of a data-driven algorithm from [15] in 


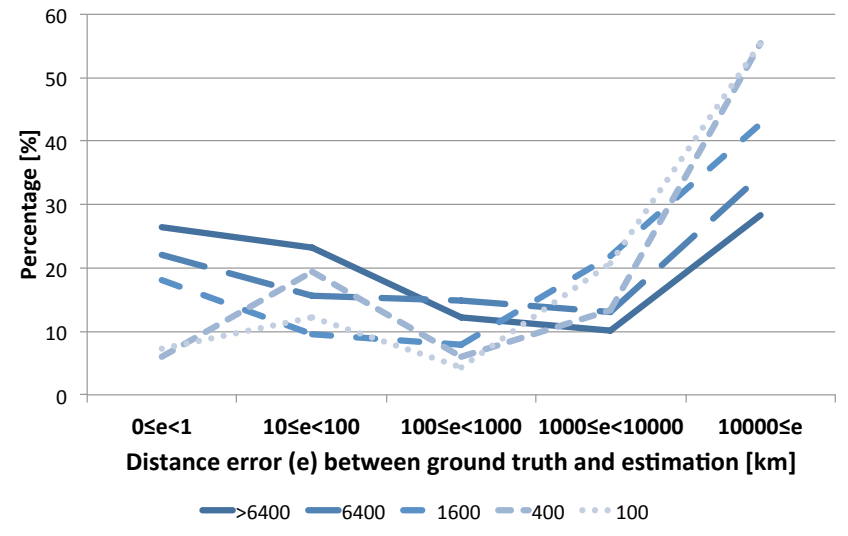

Figure 3. Comparison of the performance of a data-driven algorithm [15] on grids with different training data density. Query video from a denser area has higher chance of being estimated with lower error in distance.

different regions with varying data densities. The world map was divided into 64,800 grids of one latitude by one longitude each and the number of training data in each grid was counted. Fig. 3 shows the performance of the algorithm for different data densities. The different curves are for different values of the training data density, i.e., we look at the performance in grids with varying quantities of videos: over 6400, 6400, 1600, 400, and 100. The x-axis corresponds to the different error ranges in $\mathrm{km}$ and the $y$-axis to the percentage of geo-tagged videos in these error ranges. Grids with a denser population of training data perform significantly better than those with lesser training data. Thus, estimation models must be developed to handle data sparsity.

One can exploit the fact that while the training data may be small, test data may be very large. Existing algorithms do not take this into account. Thus the question of interest is, "Can we intelligently process the test/query videos in such a way that each additional query video not only is placed but also improves the quality of the existing database?" To take a simple example demonstrating our idea, let us suppose that we have two query videos, Q1 and Q2, with associated textual tags $\{$ berkeley, sathergate, california\} and ssathergate, california $\}$ respectively. Assume that the training set only contains the tags, \{berkeley, california\}. If Q1 and Q2 were to be processed independently, then Q1's location estimation would be good whereas the location ambiguity of Q2 would be much larger. However, if we jointly process Q1 and Q2, then given that Q1 and Q2 have the tag "sathergate" in common, it is very likely that their locations are also very close by and hence an intelligent algorithm would estimate their locations to be the same, which would improve the location accuracy of Q2. The proposed graphical model framework in the next section applies this principle to a database of query videos and appropriately weighs the edges based on the common tags between the videos.

\section{GRAPHICAL MODELS FOR GEO-TAGGING}

Graphical models provide an efficient representation of dependencies amongst different random variables and have been extensively studied in the statistical learning theory community [17]. The random variables in our setup are the geo-locations of the query videos that need to be estimated. We treat the textual tags and visual and audio features as observed random variables that are probabilistically related to the geo-location of that video. The goal is to obtain the best estimate of the unobserved random variables (locations of the query videos) given all the observed variables. We use graphical models to characterize the dependencies amongst the different random variables and use efficient message-passing algorithms to obtain the desired estimates. We give a brief introduction to graphical models and apply the framework to our setup.

An undirected graphical model or a Markov Random Field (MRF) $G(V, E)$ consists of a vertex set $V$ and an edge set $E$. The vertices (nodes) of the graph represent random variables $\left\{x_{v}\right\}_{v \in V}$ and the edges capture the conditional independencies amongst the random variables through graph separation [17]. The joint probability distribution for a $N$ node pairwise MRF can be written as follows [17],

$$
p\left(x_{1}, \ldots, x_{N}\right)=\prod_{i \in V} \psi\left(x_{i}\right) \prod_{(i, j) \in E} \psi\left(x_{i}, x_{j}\right) .
$$

$\psi($.$) 's are known as potential functions that depend on the$ probability distribution of the random variables. A typical problem of inference over a graphical model involves finding the marginal distribution of the random variables $p\left(x_{i}\right)$. Finding the exact marginals is in general an NP-hard problem [17] and approximation algorithms such as the sumproduct algorithm are used in practice. In the sum-product algorithm, messages are passed between nodes that take the following form:

$$
m_{j \rightarrow i}\left(x_{i}\right) \propto \int_{x_{j}} \psi\left(x_{i}, x_{j}\right) \psi\left(x_{j}\right) \prod_{k \in N(j) / i} m_{k \rightarrow j}\left(x_{j}\right) d x_{j},
$$

where $m_{j \rightarrow i}\left(x_{i}\right)$ is the message passed from node $j$ to node $i$ and $N(j)$ is the set of neighbors of $j$. The messages are iteratively passed until convergence and the final estimate of $p\left(x_{i}\right)$ is obtained as follows, $\hat{p}\left(x_{i}\right) \propto \psi\left(x_{i}\right) \prod_{j \in N(i)} m_{j \rightarrow i}\left(x_{i}\right)$. This algorithm is seen to work well in practice for many applications.

In order to obtain a graphical model representation for our problem setup, we need to model the joint distribution of the query video locations given the observed data. Since it is hard to obtain the exact probability distribution, we will use a simplistic conditional dependency model for the random variables as described below. Each node in our graphical model corresponds to a query video and 
the associated random variable is the geo-location of that query video (i.e., latitude and longitude). Intuitively, if two images are nearby, then they should be connected by an edge since their locations are highly correlated. The problem is that we do not know the geo-locations a priori. However, given that textual tags are strongly correlated to the geo-locations, a common textual tag between two images is a good indication of the proximity of geo-locations. Hence, we will build the graphical model by having an edge between two nodes if and only if the two query videos have at least one common textual tag. Note that this textual tag need not appear in the training set. Fig. 4 shows an example of one such graph. The edge potential functions appropriately weigh the edge based on the common textual tag. The model could be further improved using the audio and visual features as well.

Let $x_{i}$ be the geo-location of the $i$ th video and $\left\{t_{i}^{k}\right\}_{k=1}^{n_{i}}$ be the set of $n_{i}$ tags associated with this video. Based on our model, under a pairwise MRF assumption, the joint probability distribution factorizes as follows:

$$
\begin{aligned}
p\left(x_{1}, \ldots, x_{N} \mid\left\{t_{1}^{k}\right\}, \ldots .,\left\{t_{N}^{k}\right\}\right) \propto & \prod_{i \in V} \psi\left(x_{i} \mid\left\{t_{i}^{k}\right\}\right) \\
& \prod_{(i, j) \in E} \psi\left(x_{i}, x_{j} \mid\left\{t_{i}^{k}\right\},\left\{t_{j}^{k}\right\}\right) .
\end{aligned}
$$

Given the potential functions, one could use the sumproduct iterates (2), to estimate $p\left(x_{i} \mid\left\{t_{1}^{k}\right\}, \ldots \ldots,\left\{t_{N}^{k}\right\}\right)$.

We now need to model the node and edge potential functions. The literature provides numerous techniques for modeling potential functions and adaptively learning them from the given data [18]. We use the following simple model for the potential functions. Given the training data, we fit a Gaussian Mixture Model (GMM) for the distribution of the location given a particular tag $t$, i.e., $p(x \mid t)$. The intuition is that tags usually correspond to one or more specific locations and the distribution is multi-modal (e.g., the tag "washington" can refer to two geographic places). To estimate the parameters of the GMM, we use an algorithm based on Expectation Maximization [19] that adaptively chooses the number of components for different tags using a likelihood criterion. Assuming conditional independence for different tags, we take the node potential as follows, $\psi\left(x_{i}\right) \propto \prod_{k=1}^{n_{i}} p\left(x_{i} \mid t_{i}^{k}\right)$. For the potential functions, $\psi\left(x_{i}, x_{j} \mid\left\{t_{i}^{k}\right\},\left\{t_{j}^{k}\right\}\right)$, we use a very simple model. Intuitively, if the common tag between two query videos $i$ and $j$ occurs too frequently either in the test set or the training set, that tag is most likely a common word like "video" or "photo" which does not really encode any information about the geographic closeness of the two videos. In this case, we assume that the edge potential is zero (drop edge $(i, j)$ ) whenever the number of occurrences of the tag is above a threshold. When the occurrence of the common tag is less frequent, then it is most likely that the

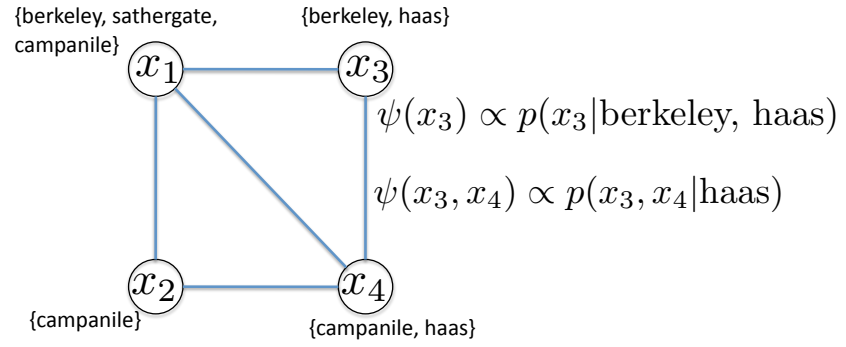

Figure 4. An example graphical model for geo-tagging. geographic locations are very close to each other and we model the potential function as an indicator function,

$$
\psi\left(x_{i}, x_{j} \mid\left\{t_{i}^{k}\right\},\left\{t_{j}^{k}\right\}\right)=\left\{\begin{array}{cc}
1 & \text { if } x_{i}=x_{j} \\
0 & \text { otherwise. }
\end{array}\right.
$$

This model is a hard-threshold model and we can clearly use a soft-version wherein the weights on the edges for the potential functions are appropriately chosen.

Further, we propose the following simplification, which leads to analytically tractable expressions for the potential functions and message updates. Given that for many of the tags, the GMM will have one strong mixture component, the distribution $\psi\left(x_{i}\right)$, can be approximated by a Gaussian distribution with the mean $\left(\tilde{\mu}_{i}\right)$ and variance $\left(\tilde{\sigma}_{i}^{2}\right)$ given by,

$$
\left(\tilde{\mu}_{i}, \tilde{\sigma}_{i}^{2}\right)=\left(\frac{\sum_{k=1}^{n_{i}} \frac{1}{\sigma_{i}^{k 2}} \mu_{i}^{k}}{\sum_{k=1}^{n_{i}} \frac{1}{\sigma_{i}^{k 2}}}, \frac{1}{\sum_{k=1}^{n_{i}} \frac{1}{\sigma_{i}^{k 2}}}\right),
$$

where $\mu_{i}^{k}$ and $\sigma_{i}^{k 2}$ are the mean and variance of the mixture component with the largest weight of the distribution $p\left(x_{i} \mid t_{i}^{k}\right)$. Under this assumption, the iterations of the sumproduct algorithm take on the following simplistic form. Node $i$ at iteration $m$, updates its location estimate $\left(\hat{\mu}_{i}(m)\right)$ and variance $\left(\hat{\sigma}_{i}^{2}(m)\right)$ as follows,

$$
\begin{aligned}
& \hat{\mu}_{i}(m)= \frac{\frac{1}{\tilde{\sigma}_{i}^{2}} \tilde{\mu}_{i}+\sum_{j \in N(i)} \frac{1}{\hat{\sigma}_{j}^{2}(m-1)} \hat{\mu}_{j}(m-1)}{\frac{1}{\tilde{\sigma}_{i}^{2}}+\sum_{j \in N(i)} \frac{1}{\hat{\sigma}_{j}^{2}(m-1)}}, \\
& \hat{\sigma}_{i}^{2}(m)=\frac{1}{\frac{1}{\tilde{\sigma}_{i}^{2}}+\sum_{j \in N(i)} \frac{1}{\hat{\sigma}_{j}^{2}(m-1)}}
\end{aligned}
$$

The location estimate for the $i$ th query video $\hat{x}_{i}$ is taken to be $\hat{\mu}_{i}(m)$ at the end of $m$ iterations, or when the algorithm has converged. The variance $\hat{\sigma}_{i}^{2}(m)$ provides a confidence metric on the location estimate. Fig. 5 provides an illustration of the algorithm.

Given the graphical model framework, it is now easy to incorporate visual and audio features. These features can 


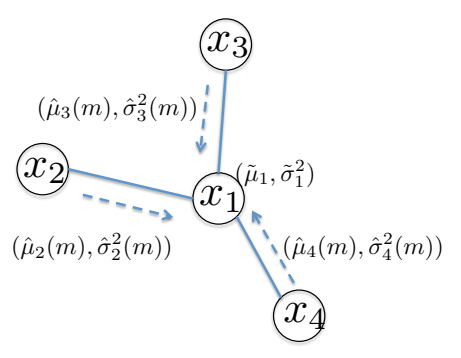

Figure 5. Illustration of messages passed along the edges.

be used to modify the potential functions $\psi\left(x_{i}, x_{j}\right)$ on the edges. The intuition is that if two images are similar in some feature space, then they are also most likely geographically closer. However, this intuition holds true only when we already have a coarse estimate of their locations and we want to further refine it. For this purpose, we adopt a hierarchical approach. We first obtain a coarse estimate of the query video's location using only the tags in the graphical model. We then choose a subgraph for each query video consisting only of query and training videos within some particular radius of each other. Visual and acoustic features are obtained for each video using GIST and MFCC features similar to what Friedland et al. use in [16]. The probability distribution of the geographic distance between two videos given the closeness of the videos in the visual and audio feature space is modeled as a mixture of exponentials and is incorporated in the edge potential function $\psi\left(x_{i}, x_{j}\right)$.

\section{EXPERIMENTAL RESULTS}

We tested our algorithm on the MediaEval 2011 Placing Task data set, which consists of Creative Common-licensed videos manually crawled from Flickr [20]. Videos are not filtered or selected in any way to make the data set more relevant to the task, and are therefore likely representative of videos selected at random. In current online databases (e.g., Flickr and YouTube), videos are not equally distributed over the earth (Fig. 2), so downloading a random sample shows a large bias for certain locations. The metadata for each video includes user-contributed title, tags, and description, and may include other data as well. Flickr requires that an uploaded video be created by its uploader and therefore many videos are home-video style short clips. Manual inspection of the data set led us initially to conclude that most of visual/audio contents lack reasonable evidence to estimate the location without textual metadata. For example, unlike the filtered test datasets used in [6], many videos were recorded indoors or in a private space such as the backyard of a house. The data set consists of 10216 training videos and 5347 query videos.

Performance evaluation is carried out for different values of training and test data. Fig. 3 shows the performance of [15]. We evaluate the performance of the proposed algorithm for different subsets of the training videos in comparison with this system. In order to understand the

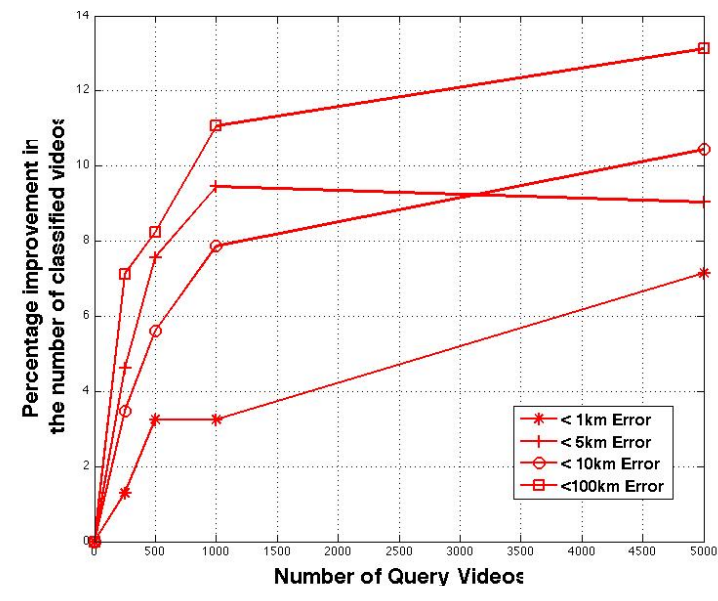

Figure 6. Performance improvement in geo-tagging 5347 videos using a training set of 500 videos as a function of the number of query videos used in the graphical model.

performance improvements obtained in the data sparse case, we use 500 training videos and plot the performance improvement as more and more query videos are added to the system. Fig. 6 shows the performance improvement in different categories. The number of test videos for this plot is fixed at 5347. The $\mathrm{x}$-axis shows the number of query videos that were used in forming the graph. For example, the point 1000 on the $\mathrm{x}$-axis means that, while building the graph for all the 5347 videos, each video had a neighbor only from these 1000 videos. The best case is when any video in 5347 videos could be a neighbor of any other video. The y-axis is the performance improvement over the baseline system. The different curves correspond to different error categories i.e. $<1 \mathrm{~km}$ error, $10 \mathrm{~km}-100 \mathrm{~km}$ error etc. The performance improvement in each category is calculated as follows,

$$
A=\text { Num. of videos with }<1 k m \text { error using our alg, }
$$

$B=$ Num. of videos with $<1 \mathrm{~km}$ error using the baseline alg, Perf. improvement in $<1 \mathrm{~km}$ error category $=\frac{A-B}{B} \times 100$.

This extends to the other error categories. The performance improvement can be over $10 \%$ and increases with the number of test videos. However, one can clearly see the diminishing returns with increasing number of query videos.

Fig. 7 shows the performance improvement from the test videos as the number of training videos increases. In this plot, the underlying graph was generated with all the 5347 test videos and the performance improvement was observed as a function of the number of training videos. Though there are gains initially, as the number of training videos increases, the performance improvement obtained by using the query videos decreases. This is to illustrate that in the sparse data case with fewer training videos, the performance improvements can be large. However, with a larger training set, the performance improvement can be very marginal. In practice, given that most of the 


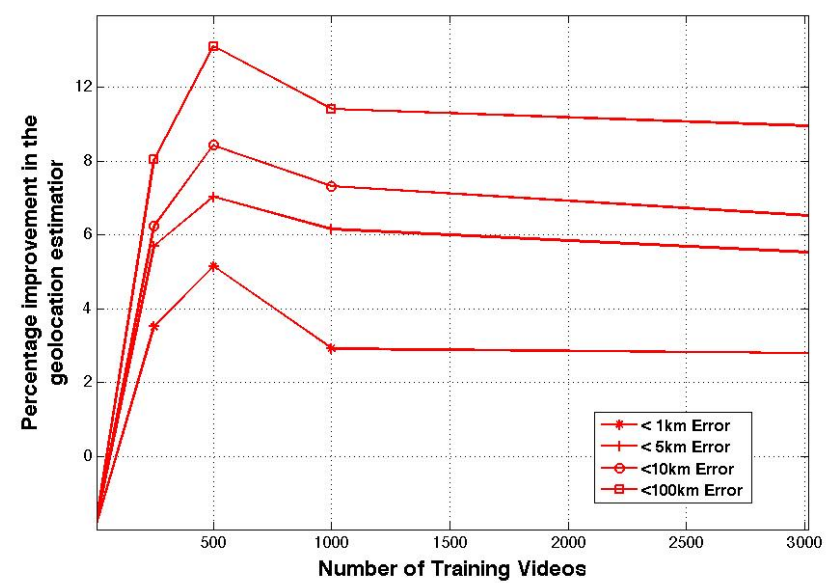

Figure 7. Performance improvement in geo-tagging 5347 videos as a function of the number of training videos.

videos are not geo-tagged, i.e., the test set can be orders of magnitude larger than the training set, one can hope to achieve significant performance gains.

\section{CONCLUSIONS AND FUTURE WORK}

We presented a novel approach to address the problem of geo-tagging multimedia content on the Web like Flickr videos and images. In particular, we addressed the case where the training data set is sparse and explored the possibility of using the test data set to improve the quality of the training database. We proposed a graphical model framework, posed the problem of geo-tagging as one of inference over this graph, and showed that performance improvements can be achieved by processing the test data set in an intelligent way.

Various issues remain to be explored. The modeling of edge potentials in the graphical model is very naive and one can explore richer models such as hierarchical models (e.g., latent dirichlet topic models) to model the correlations on the edges. The node potentials are further modeled as a product of the distributions given each tag individually. However, the distribution of the locations given multiple tags is not independent. For example, the location distributions of the tags "berkeley" and "sathergate" are clearly not independent. Hence a better correlation model needs to be explored for these distributions.

\section{REFERENCES}

[1] "Mediaeval web site," http://www.multimediaeval.org.

[2] M. Larson, M. Soleymani, P. Serdyukov, S. Rudinac, C. Wartena, V. Murdock, G. Friedland, R. Ordelman, and Gareth J.F. Jones, "Automatic Tagging and Geo-Tagging in Video Collections and Communities," in ACM International Conference on Multimedia Retrieval (ICMR 2011), April 2011, p. to appear.

[3] G. Friedland, O. Vinyals, and T. Darrell, "Multimodal Location Estimation," in Proceedings of ACM Multimedia, 2010, pp. 1245-1251.
[4] G. Schindler, M. Brown, and R. Szeliski, "City-scale location recognition," in IEEE Conference on Computer Vision and Pattern Recognition, 2007. CVPR'07, 2007, pp. 1-7.

[5] W. Zhang and J. Kosecka, "Image based localization in urban environments," in 3D Data Processing, Visualization, and Transmission, 3rd Intl. Symposium on, 2006, pp. 33-40.

[6] J. Hays and A.A. Efros, "IM2GPS: estimating geographic information from a single image," in IEEE Conference on Computer Vision and Pattern Recognition, 2008. CVPR 2008, 2008, pp. 1-8.

[7] J. Luo, D. Joshi, J. Yu, and A. Gallagher, "Geotagging in multimedia and computer vision-a survey," Multimedia Tools Appl., vol. 51, pp. 187-211, Jan. 2011.

[8] T. Rattenbury and M. Naaman, "Methods for extracting place semantics from Flickr tags," ACM Transactions on the Web (TWEB), vol. 3, no. 1, 2009.

[9] P. Serdyukov, V. Murdock, and R. van Zwol, "Placing Flickr photos on a map," in ACM SIGIR, 2009, pp. 484-491.

[10] O. Van Laere, S. Schockaert, and B. Dhoedt, "Ghent university at the 2011 placing task," in Proc. of MediaEval, 2011

[11] L. Cao, J. Yu, J. Luo, and T. Huang, "Enhancing semantic and geographic annotation of web images via logistic canonical correlation regression," in Proceedings of the 17th ACM international conference on Multimedia, New York, NY, USA, 2009, MM '09, pp. 125-134, ACM.

[12] A. Gallagher, D. Joshi, J. Yu, and J. Luo, "Geo-location inference from image content and user tags," in Proceedings of IEEE CVPR. 2009, IEEE.

[13] David J. Crandall, Lars Backstrom, Daniel Huttenlocher, and Jon Kleinberg, "Mapping the world's photos," in Proc. of $W W W$ '09, New York, NY, USA, 2009, pp. 761-770, ACM.

[14] P. Kelm, S. Schmiedeke, and T. Sikora, "A hierarchical, multimodal approach for placing videos on the map using millions of flickr photographs," in Proc. of SBNMA '11, New York, NY, USA, 2011, pp. 15-20, ACM.

[15] G. Friedland, J. Choi, H. Lei, and A. Janin, "Multimodal Location Estimation on Flickr Videos," in Proc. of the 2011 ACM Workshop on Social Media, Scottsdale, Arizona, USA, 2011, pp. 23-28, ACM.

[16] J. Choi, H. Lei, and G. Friedland, "The 2011 ICSI Video Location Estimation System," in Proc. of MediaEval, 2011.

[17] M.J. Wainwright and M.I. Jordan, "Graphical models, exponential families, and variational inference," Foundations and Trends $\mathrm{R}$ in Machine Learning, vol. 1, pp. 1-305, 2008.

[18] L. Song, A. Gretton, D. Bickson, Y. Low, and C. Guestrin, "Kernel belief propagation," Preprint arXiv:1105.5592, 2011.

[19] N. Vlassis and A. Likas, "A greedy em algorithm for gaussian mixture learning," Neural Processing Letters, vol. 15, no. 1, pp. 77-87, 2002.

[20] A. Rae, V. Murdock, and P. Serdyukov, "Working Notes for the Placing Task at MediaEval 2011," in MediaEval 2011 Workshop, Pisa, Italy, September 2011. 